\title{
Agôn
}

Revue des arts de la scène

HS 1 | 2011

Mettre en scène l'événement

\section{Une expérience de théâtre témoin}

Everything's Back to Normal in New York City: Below Canal, a Work in Progress

Ariane Zaytzeff

\section{OpenEdition}

Journals

Édition électronique

URL : http://journals.openedition.org/agon/1772

DOI : 10.4000/agon. 1772

ISSN : 1961-8581

Éditeur

Association Agôn

Référence électronique

Ariane Zaytzeff, «Une expérience de théâtre témoin », Agôn [En ligne], HS 1 | 2011, mis en ligne le 05 septembre 2011, consulté le 17 avril 2020. URL : http://journals.openedition.org/agon/1772 ; DOI : https://doi.org/10.4000/agon.1772 


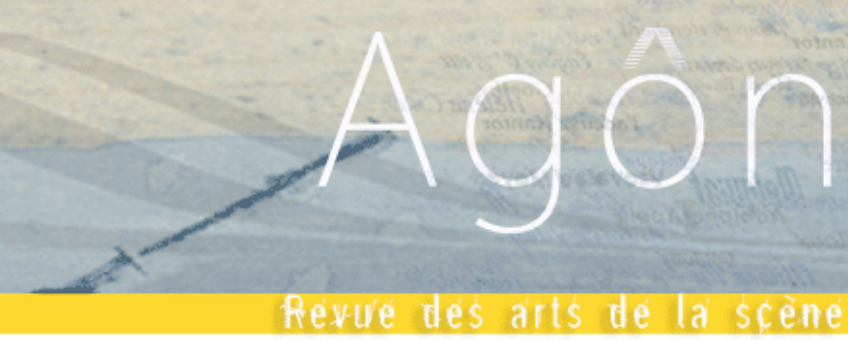

\title{
Une expérience de théâtre témoin
}

\author{
Everything's Back to Normal in New York City : Below Canal, a Work in Progress. \\ ARIANE ZAYTZEFF
}

Au lendemain des attentats du 11 septembre 2001, le gouvernement américain cherche une réponse à la mesure de la catastrophe, qui s'avère dévastatrice à l'échelle locale, nationale et internationale. Il s'agit non seulement de remédier aux dégâts matériels et humains mais aussi de réaffirmer la capacité du pays à se défendre et rétablir la puissance mise en danger, et la stratégie adoptée par George W. Bush est l'attaque et l'action. Alors que les débats politiques se déploient sur la scène nationale et internationale, il devient clair que les tactiques se dessinent au détriment du local. Après avoir proclamé le 14 septembre journée nationale de prière et de mémoire pour les victimes des attaques terroristes du 11 septembre $2001^{1}$, George W. Bush prononce trois jours plus tard un discours sur la réponse militaire des États-Unis. Dans ce discours, il déclare : " Aujourd'hui, des millions d'Américains ont fait leur deuil et prié, et demain nous nous remettons au travail ${ }^{2}$. » Cette déclaration, reprise plus loin dans son discours, montre clairement une volonté d'avancer et redevenir actif. Le discours 16 septembre 2001 marque un tournant dans la réaction des États-Unis face aux attentats. Après cinq jours seulement, le gouvernement met fin au deuil pour passer à une autre étape, celle de la guerre contre la terreur, qui a pris les dimensions que l'on connaît.

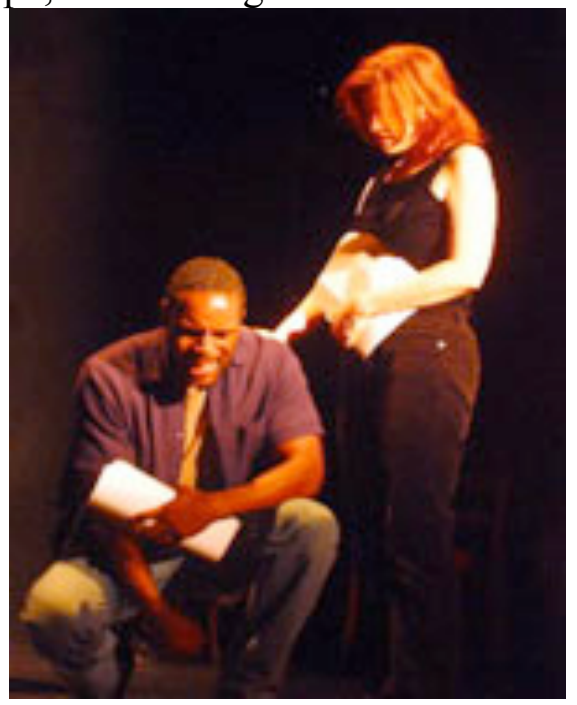

Pris au cœur de cet engouement pour l'action et les représailles, les New-Yorkais, et en particulier les habitants de Downtown Manhattan, ont peine à trouver leur place. En effet, il faudra bien plus de cinq jours pour rendre le quartier à nouveau accessible, les dégâts causés par la chute des tours étant très lourds. La population de tout un quartier de Manhattan est déplacée et dans une situation précaire. Bien que des plans d'urgence de relogement et des écoles temporaires soient mis en place, la communauté de Downtown a perdu ses repères et chacun se retrouve isolé. Diana Taylor,

\footnotetext{
${ }^{1}$ George W. Bush, « National Day of Prayer and Remembrance for the Victims of the Terrorist Attacks on September 11, 2001, » url=http://www.whitehouse.gov/news/releases/2001/09/20010913-7.html, consulté le 25/05/2008.

2 George W. Bush, «Remarks Upon Arrival, » url=http://www.whitehouse.gov/news/releases/2001/09/200109162.html, consulté le 25/05/2008.
} 
professeur de performance studies à New York University, décrit ainsi sa première réaction : « Nous nous tenions là, paralysés, regardant, témoins sans narration, membres d'un chœur tragique qui trébuchait sur la mauvaise scène ${ }^{3}$. » La place de témoin est celle qui incombait naturellement à ceux qui ont vécu l'évènement, l'ont subi et l'ont vu de leurs yeux. Ils font partie de la catastrophe, ils sont des superstes qui peuvent témoigner du 11 septembre parce qu'ils l'ont traversé et lui ont survécu. Mais dès le jour des attaques, les rôles avaient déjà été distribués :

Si cela était une tragédie, nous n'étions pas reconnus comme des participants. Le rôle de témoin, participant responsable et éthique plutôt que spectateur de la crise, s'effondrait dans les décombres des discours sur les héros, les victimes, et le reste : nous. [...] il n’y avait pas de place pour nous, pas de participation qui puisse être signifiante ${ }^{4}$.

Cette distribution des rôles a pour effet d'exclure les New-Yorkais et participe à une «crisis of witnessing $^{5} \gg$ (crise de témoignage). Diana Taylor souligne que, bien que le 11 septembre 2001 soit appelé une tragédie, il ne s'agit pas d'une tragédie aristotélicienne qui obéirait à une logique organique et à l'issue de laquelle le héros ainsi que le public parviendraient à une reconnaissance ou une catharsis; le public est tout simplement exclu de la représentation ${ }^{6}$. Ce rapport à la tragédie est en lien direct avec la question du témoin. En effet, la tragédie grecque ne fait pas que présenter une variété d'évènements terribles, elle nomme aussi une structure entière de pensée, elle construit du sens, un système qui compose une éthique. Marc Nichanian appelle la tragédie la «politique du témoin $^{7}$ », car elle rend compte à la fois d'un évènement et de l'impossibilité d'en rendre compte. Pour lui, cette politique n'est possible que dans le contexte de la Grèce antique et nous avons perdu la politique du témoin parce que nous n'avons plus d'espace, nulle part, pour la faire advenir. Le traitement du 11 septembre et de ses conséquences en est un bon exemple. Les espaces de parole publics sont occupés par les discours politiques et patriotiques, et la difficulté, voire l'impossibilité, pour les gens qui ont vécu cet évènement, de trouver les mots adéquats pour décrire, exprimer, questionner leurs expériences n'est pas prise en compte. Le système narratif qui se met en place est faussement sûr de lui afin de servir de base à un élan combatif qui ne tient pas compte de la voix du chœur. La crise de témoignage est donc non seulement provoquée par l'ampleur de l'évènement, qui fait du témoignage par récit ou représentation un réel défi, mais aussi par l'imposition d'une ligne narrative monochrome qui nie la présence de ce défi. En réponse directe à cette crise, on trouve souvent dans les créations théâtrales imaginées après le 11 septembre un désir de ré-appropriation de l'évènement, qui passe par la réintégration du chœur. Par la volonté de donner voix à ceux qui ont vécu la «tragédie », certaines pièces de théâtre initient un retour aux origines : les hommes qui font la cité où l'évènement s'est produit; le chœur qui est l'origine du théâtre et de la tragédie, bien avant que le protagoniste n'apparaisse. Citons par exemple le September $11^{\text {th }}$ Testimony Project, qui se concentre sur les survivants des attentats ; After Images, qui réunit les performances de trentetrois artistes de différentes disciplines qui offrent leurs réflexions sur l'impact du 11 septembre sur leurs vies personnelles et artistiques; Our True Colours, qui met en scène les textes d'élèves de lycées de New York City (Manhattan, Brooklyn, Staten Island, Bronx et Queens) et Return to the Upright Position, un témoignage écrit dans les six mois qui ont suivi les attentats par quatorze écrivains et artistes, compilé par Caridad Svich.

\footnotetext{
3 Diana Taylor, "Lost in the field of vision : Witnessing September 11 » in The Archive and the Repertoire : Performing Cultural Memory in the Americas, Durham, Duke University Press, 2003, p. 237.

${ }^{4} I d$., p. 243 .

${ }^{5}$ Shoshana Felman et Dori Laub utilisent cette expression dans leur analyse de la littérature et psychanalyse après l'Holocauste. S. Felman et D. Laub, Testimony : Crises of Witnessing in Literature, Psychoanalysis and History, New York, Routledge, 1991.

${ }^{6}$ Diana Taylor, op. cit., p. 261.

${ }^{7}$ Marc Nichanian, "Catastrophic Mourning » in Loss: The Politics of Mourning, D.L. Eng et D. Kazanjian (ed.), Berkeley, University of California Press, 2003, p.140.
} 


\section{Naissance du projet de théâtre témoin}

C'est à la crise de témoignage que la pièce que je veux évoquer ici répond. Jack Saul, psychologue et directeur du programme d'études internationales du traumatisme à New York $\left(\right.$ ITSP $\left.^{8}\right)$, habite dans un des quartiers du sud de Manhattan, et il observe que

[1]es méthodologies et les institutions qui répondent à ceux qui ont besoin d'aide après un trauma psychosocial massif portent souvent peu d'attention à la dimension sociale de la souffrance, et encore moins aux besoins, aux voix et aux capacités de guérison naturelles des personnes affectées ${ }^{9}$.

Il assiste à la mise en place d'un soutien psychologique qui se concentre sur la détection du syndrome de stress post-traumatique, ou PTSD, en particulier chez les enfants. Face à cette prise en charge qui se focalise sur l'individu au détriment des ressources déjà présentes dans la communauté, il crée le Downtown Community Resource Center ${ }^{10}$ afin de mettre à disposition des habitants de Downtown New York un espace où ils peuvent être acteurs de leur propre reconstruction. Le DCRC développe deux projets en parallèle : le projet d'archives vidéo permet aux habitants du quartier de venir témoigner de leurs expériences du 11 septembre et projette ensuite ces vidéos dans des lieux publics et les met à disposition en ligne. L'autre projet est la création d'une pièce de théâtre à partir d'interviews avec une dizaine d'habitants du sud de Manhattan. Le but de ces deux initiatives est de permettre aux habitants de partager leurs histoires dans leurs termes, à leur rythme, et avec un sentiment d'écoute et réception de la part de leur communauté. L'intégration du théâtre dans cette volonté de se concentrer sur le local et la communauté est une évidence pour Jack Saul, qui utilise déjà le théâtre dans son travail quotidien. En effet, dans le cadre de l'ITSP, il a créé avec Steven Reisner, psychologue, acteur et metteur en scène, et Robert Gourp, metteur en scène, la troupe Theater Arts Against Political Violence ou TAAPV (Arts du Théâtre contre la Violence Politique). La troupe a commencé à travailler avec des survivants de torture puis des communautés de réfugiés installés à New York, et a affiné au cours des années une technique de travail que Jack Saul appelle « theater of witness » (théâtre témoin). À l'invitation d'une communauté, ils en interviewent certains membres puis improvisent des scènes à partir de leurs récits. Les individus interviewés peuvent rester et réagir, commenter, proposer des modifications, et le travail continue. Les acteurs intègrent leurs propres réactions et histoires à la pièce, puis la jouent devant la communauté en question. Des discussions avec le public ont lieu après les représentations, et à partir de ces échanges, la pièce est retravaillée et de nouveaux éléments sont incorporés pour les représentations à venir ${ }^{11}$. On voit que cette méthode emprunte des éléments à des pratiques théâtrales comme celle du psychodrame de Moreno au début du $\mathrm{XX}^{\mathrm{e}}$ siècle et, plus récemment, le théâtre de l'opprimé d'Augusto Boal ${ }^{12}$. Lorsque les membres de la communauté sont présents en répétition, ils ne sont pas confinés au rôle de spectateurs mais peuvent intervenir dans le déroulement du scénario. Cependant, lors des représentations, seuls les acteurs professionnels jouent sur scène, et le public n'intervient qu'après le spectacle pour offrir ses réflexions et conseils, ce qui diffère de l'art-thérapie ou de la pratique de Boal.

Fort de cette méthode en perpétuelle évolution, Jack Saul imagine donc une réponse artistique et collective aux attentats du 11 septembre. Le nom de théâtre témoin n'a pas été choisi au hasard, et il semble convenir parfaitement à la crise de témoignage provoquée par l'évènement. Il s'agit de rendre compte de la diversité des expériences en accueillant des récits autres que ceux offerts et répétés par les médias, des récits qui prennent en compte et naissent de l'impossibilité de dire. Le projet peut se lire comme une reconquête de la parole au niveau local, une valorisation du détail et du particulier, en résistance à la confiscation de l'évènement à des fins politiques. L'un des

\footnotetext{
${ }^{8}$ http://itspnyc.org

9 Jack Saul, « Promoting Community Resilience in Lower Manhattan After September 11, 2001, » in AFTA Monograph Series, Winter 2007, p. 69.

${ }^{10} \mathrm{http}: / /$ itspnyc.org/programs-downtown.htm

11 Jack Saul, "International Trauma Studies Program», in The New Humanitarians : Inspiration, Innovation and Blueprints for Visionaries, Chris E.Stout (ed.), Londres, Praeger, 2008, p. 215.

${ }^{12}$ Augusto Boal, Theatre of the Oppressed. Londres, Pluto Press, 1979.
} 
personnages de la pièce, le professeur Sparkle, souligne le sentiment d'inutilité des survivants lorsqu'ils racontent leur histoire, et ce quel que soit l'interlocuteur. Rien n'est fait de leurs histoires : elles sont peut-être stockées quelque part pour servir un jour, ou plutôt pour pouvoir être oubliées. Un personnage pense qu'il est inutile de parler encore car tout a été dit, un autre se demande pourquoi son histoire pourrait encore susciter de l'intérêt alors que tant d'autres ont été racontées. En réponse à ces doutes, une jeune femme encourage chacun à parler et partager son histoire car c'est une façon de se toucher les uns les autres : cela rend l'autre réel. Pour Steven Reisner, le théâtre permet d'

admettre la déchirure du tissu social, [de] faire le deuil de la tragédie, et [de] tenter de transformer l'expérience en une réorganisation de la structure culturelle afin d'inclure une reconnaissance plus humble des limites, de la mortalité et de la compassion humaines ${ }^{13}$.

Ainsi, bien que Marc Nichanian ne croie pas que le théâtre puisse encore être le lieu de la politique du témoin ${ }^{14}$, c'est ce que la troupe du DCRC s'efforce de faire. Répondre aux besoins de la communauté passe par la mise en place d'une structure de sens au sein de laquelle les individus retrouvent un sentiment d'appartenance et peuvent reconnaître leur vulnérabilité. Cependant, la structure théâtrale imaginée par la troupe n'est pas figée et se caractérise par son adaptabilité. Du processus de création jusqu'aux représentations, elle prend la forme d'une recherche : de méthode, de témoins, de mots, d'images, de sons, et de sens.

Lorsque l'idée du projet théâtral naît, Jack Saul fait appel à Abigail Gampel. Celle-ci est metteur en scène et fait partie du groupe New Yorkers Say No to War (NYSNW), un groupe d'artistes, activistes et professeurs qui protestent contre la guerre en Irak et ont créé un cabaret à cet effet. Quand il lui propose de se joindre au projet théâtre du DCRC, il y a tout à faire : trouver les acteurs, les gens à interviewer, écrire la pièce et même trouver une méthode de travail. Le groupe se forme avec des acteurs de la troupe TAAPV, des membres de NYSNW, et Abigail Gampel et Jack Saul organisent des auditions pour arriver à un total de huit acteurs : Carolyn Baeumler, Chad Coleman, Chris Cuomo, Christine Farrell, Jennifer Odishoo, Esther Perel, Steven Rattazzi et Steven Reisner. Tous sont New-Yorkais et ils s'engagent ensemble dans un travail où ils sont comédiens, écrivains, et conducteurs d'entretiens, et pour lequel ils ré-explorent leurs propres expériences du 11 septembre. Pour ce qui est du choix des personnes à interviewer, Jack Saul, du fait de son travail préalable avec diverses communautés, est en contact avec un grand nombre d'individus aux histoires variées. Cependant très vite, Abigail Gampel et les acteurs prennent part au processus de sélection de façon organique, car tous sont aussi entourés de personnes qui vivent à New York et ont vécu cet évènement d'une façon ou d'une autre. Finalement, le groupe interviewe dix NewYorkais, parmi lesquels une révérende épiscopalienne qui travaillait dans les débris pour bénir ce qui était retrouvé des corps, un réfugié chilien qui avait vécu le 11 septembre 1973, deux habitants de Chinatown, et une responsable d'immeuble qui vivait à deux rues des tours jumelles. Ils s'interviewent aussi les uns les autres, et interviewent leurs proches, pour aboutir finalement à un texte conséquent.

\section{Le processus}

Le groupe improvise sa démarche au fur et à mesure des interviews, tout en gardant comme point de départ la méthode utilisée par le TAAPV. Quand je lui demande si elle pourrait qualifier la pièce de théâtre documentaire, Abigail Gampel maintient le terme de "theater of witness ». Elle convient qu'en se développant, la pièce est devenue une entité de nature hybride car la variété de méthodes de travail des comédiens ne permet pas d'y apposer un qualificatif fixe. L'un des membres du groupe travaillait dans le style d'Anna Deavere Smith, apprenant les interviews sur le bout des doigts : le moindre mot, la moindre pause, respiration, les gestes aussi. Un autre écrivait des scènes

\footnotetext{
${ }^{13}$ Steven Reisner, "Private Trauma/Public Drama : Theater as a Response to International Political Trauma", in Public Sentiments, Ann Cvetkovich et Ann Pellegrini (ed.), Special Issue of S\&F Online 2.1 (2003), url=http://www.barnard.edu/sfonline/ps/reisner.htm, consulté le 06/05/2010.

${ }^{14}$ Marc Nichanian, Ibid.
} 
à partir des interviews, un autre encore écrivait des textes sur son expérience des attentats vus de Harlem. Le travail d'écriture est le plus exigeant, et pour les acteurs, la création de la pièce est tout autant l'occasion de donner voix à la communauté du sud de Manhattan que de se donner voix à eux-mêmes. L'une des comédiennes, Chris Cuomo, n'était pas présente lors des attentats, et elle intègre dans la pièce ce besoin d'être présente à la blessure, de se sentir connectée. À ces textes autobiographiques et poétiques s'ajoutent les interviews, qui se passent dans le salon des uns et des autres ou dans des studios de théâtre loués pour l'occasion. Le groupe s'assoit en cercle avec leur invité et chacun se présente. Pendant une heure, l'invité raconte son histoire et répond aux questions des acteurs et metteur en scène. Les questions ne sont pas préparées et diffèrent selon le récit entendu. Après l'interview/témoignage, le groupe propose à l'invité de rester pour assister aux improvisations inspirées de son histoire. Certains acceptent, d'autres préfèrent partir. Parfois le groupe prend un moment pour parler de l'histoire qu'ils viennent d'entendre, d'autres fois ils commencent directement l'improvisation et démêlent leurs réactions en action. Les interviews sont filmées, comme pour le projet d'archives vidéo, et chaque acteur est responsable de la retranscription de l'une d'entre elles.

Cependant, dès le stade de l'interview, le projet de théâtre se différencie de l'archive. Lorsque huit ans plus tard Abigail Gampel me raconte certains moments des interviews, elle me donne des détails qui sont en soi très théâtraux : des sons, des images très fortes, choses à quoi le groupe est particulièrement attentif. L'écoute qu'ils offrent est propice au surgissement de détails nouveaux, précis, détachés de toute nécessité de créer un récit suivi et logique. Ainsi, une femme leur explique que l'évènement lui a rappelé la mort de sa grand-mère en Italie lors d'un bombardement pendant la Seconde Guerre Mondiale, une histoire que sa mère lui a souvent racontée. Assise dehors, sa mère et sa grand-mère nettoyaient des lentilles et la grand-mère a été touchée, laissant les lentilles se répandre sur le sol avant de tomber. Cette scène et l'expérience sonore et visuelle qu'elle offre est intégrée à la pièce.

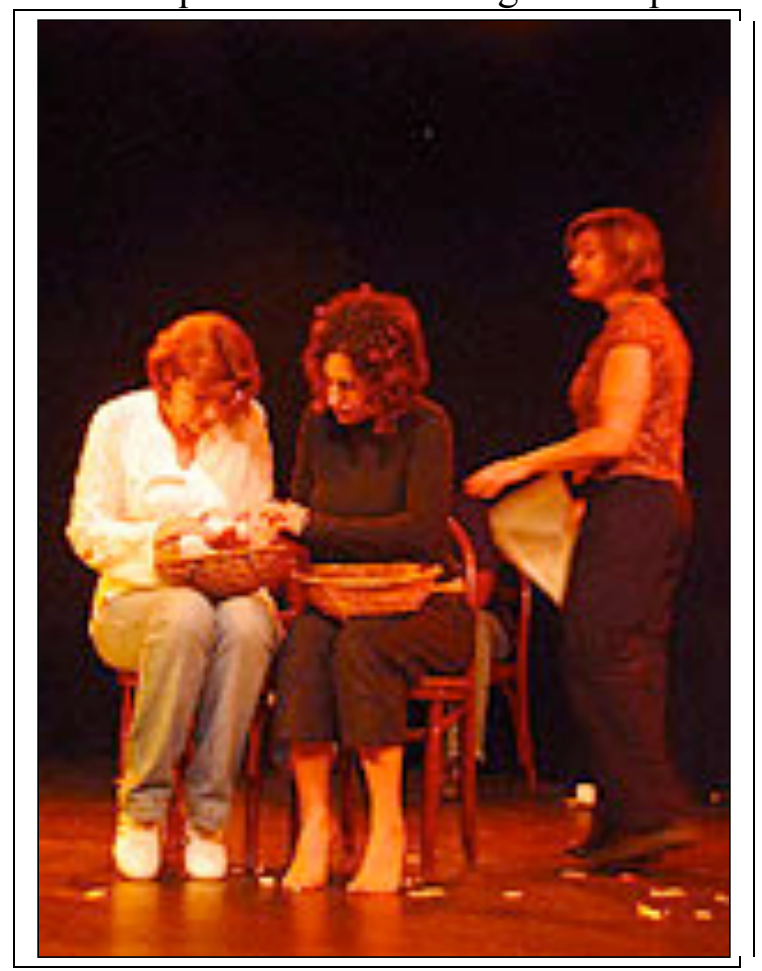

C'est ainsi qu'Abigail Gampel procède lorsqu'elle se retrouve face aux pages de retranscriptions, de textes écrits par les acteurs, et d'improvisations, pour en faire une pièce : elle saisit ce qui surgit à la relecture ou pendant une improvisation. Les acteurs eux-mêmes sentent très vite une affinité particulière avec telle ou telle personne et décident de prendre en charge le personnage né de son interview et des improvisations. Abigail Gampel se décrit comme une chef d'orchestre : il s'agissait d'être à l'écoute de la sensibilité des acteurs, saisir ce qui des interviews résonnait avec eux et ce qui résonnait ensuite ensemble. Ainsi, en voyant Steven Reisner incarner Sandro, le Chilien, en 
répétition, elle a l'idée d'en faire le narrateur, un personnage qui introduirait la pièce et servirait de fil conducteur et d'interlocuteur privilégié pour le public.

\section{La pièce}

Sandro ouvre donc la pièce avec le récit de son expérience du 11 septembre 1973, réinscrivant les attentats de 2001 dans une perspective historique. Il évoque le silence dans les rues, les avions militaires volant si près des immeubles, les bombes lâchées sur le palais présidentiel: le déroulement du coup d'État à Santiago de Chile semble étrangement similaire aux attentats de New York. Au terme de son discours, il explique que, le 11 septembre 1973, son premier réflexe a été de se réfugier chez un ami qui avait une télévision afin de regarder les images, moyen privilégié de regarder l'évènement. Un peu plus tard dans la pièce, un jeune Afro-Américain raconte son expérience personnelle du 11 septembre 2001 qui fait écho à l'expérience de Sandro. Habitant au nord dans Harlem, il s'est senti déconnecté ; il ne voyait de l'attaque que la fumée des tours au loin, il entendait les reporters à la radio, les gens dans la rue, mais cela semblait loin et irréel, il ne voyait pas. Il est donc rentré chez lui et a allumé la télé : « J'ai regardé encore et encore, attendant de sentir quelque chose mais je ne sentais rien ${ }^{15}$." Le choix du théâtre, medium vivant et par essence éphémère, pour apporter leurs témoignages revêt alors une signification de résistance, et la pièce n'hésite pas à critiquer les cadres donnés à la parole, en particulier celui offert par les médias.

DIANE. Trop de gens n'avaient nulle part où aller... Ils avaient peur. Une prise de conscience, j'ai dit « Je ne peux pas partir. » J'ai pris les choses en main! Et tout le monde était d'accord, Diane est la responsable !

Il fallait se battre pour tout.

La nourriture. Nous avions une réunion toutes les nuits. Parfois je pensais que je ne pouvais plus supplier, et puis je suis assise dans l'appartement d'une des locataires et le tapis est pollué, et elle a du mal à respirer. Elle prend sa dernière pilule pour le cœur. Il n'y avait pas moyen d'avoir des médicaments.

$[\ldots]$

Anarchie Bureaucratie

C'est comme ça, on a besoin de manger. J'ai tenu bon. J'ai supplié pour les vivants et j'ai dû défier le système. Nous étions complètement oubliés - alors que les morts - enfin... Alors voilà ma chance, en tant que vice-présidente du 40 Harrison Street, de raconter notre histoire aux médias. "Sois dans l'instant», je ne cesse de penser. John, reste avec moi... Ils vont me laisser parler de tout... Tu as un peigne ?...Je ne ressemble à rien... Flûte, mes mains sont sales, je porte ces stupides loques... J'ai l'air affreuse... Ils ne vont pas faire attention à moi. Tu peux... ah d'accord... Je dois le faire maintenant. Est-ce que je peux aller dedans pour... D'accord, je JoURNALISTE (intervenant). Non, vous êtes très bien. Prête... Lumière Caméra Action! DiANE. Oui, je suis la représentante des locataires du 40 Harrison Street. À propos des conditions de vie... Il y a des gens... Il y a tellement à dire. Ce que nous... Des personnes âgées qui... Excusez-moi, deux secondes...

Elle éclate en sanglots.

JOURNALISTE. Coupez.

DiANE. Je ne sais pas d'où c'est venu... Je suis vraiment désolée. Je vais recommencer... JOURNALISTE. C'était parfait.

DIANE. Quoi ?

JOURNALISTE. C'était parfait.

DiANE. ... mais je n'ai rien dit! Vous voulez juste me voir pleurer. C'est ça... C'est ça... C'est ça...

JOURNALISTE (intervenant entre chaque « $C^{\prime} e s t$ ça »). C'était parfait ${ }^{16}$.

La pièce ne suit pas un arc narratif uni qui formerait une histoire ou un scénario ; elle donne voix aux craintes et aux attentes de la communauté à travers le groupe d'acteurs qui prend en charge différents personnages, à l'exception de Steven Reisner qui joue uniquement Sandro. Comme il le

\footnotetext{
${ }^{15}$ Downtown Community Resource Center Theater Group, Everything's Back to Normal in New York City : Below Canal, A Work in Progress. Texte des représentations des 28, 29 et 30 septembre 2003. Extrait.

${ }^{16}$ Id.
} 
dit lors d'une de ses interventions, citant Beckett : " Il n'y a pas besoin d'une histoire, une histoire n'est pas obligatoire, juste une vie, c'est l'erreur que j'ai commise, une des erreurs, d'avoir voulu une histoire pour moi-même, alors que la vie seule suffit ${ }^{17} \gg$. En n'offrant pas un récit clair et ordonné, la pièce ne prend pas la forme d'une histoire mais cherche à offrir des bouts de vie, des extraits d'expériences. Les acteurs sont omniprésents, hormis Sandro qui se tient hors-scène lorsqu'il ne parle pas, et ils s'écoutent les uns les autres et sont amenés à interagir. Au sein du chœur de personnages, chacun a son moment, son solo.

À jardin, un écran montre des images de la ville, de fumée, parfois des extraits d'interviews. Ce dispositif ajoute au sentiment d'encombrement et de saturation de la scène, qui va grandissant au fur et à mesure que la pièce avance. Les acteurs amènent progressivement de plus en plus d'éléments qui finissent par joncher le sol. Inspirée du témoignage d'une femme qui évoquait les corps tombant des tours, les acteurs tombent les uns après les autres, se rattrapent, ou non, puis prennent des feuilles de papier qu'ils déchirent, laissant les morceaux tomber lentement sur le sol. Plus tard, lors de la scène de la mort de la grand-mère en Italie, des lentilles viennent s'éparpiller sur le sol et se mêler au papier. Lorsque la révérende raconte son expérience dans les débris et le travail des machines qui cherchaient à sortir les corps des décombres, elle se trouve au milieu de ce désordre. Enfin, l'amas de débris divers devient un autel devant lequel se recueillir. L'attention donnée à l'espace est primordiale car elle témoigne du fait que les acteurs et ceux qu'ils ont interviewés ont ressenti les effets des attentats dans leurs espaces de vie mêmes, et qu'ils abordent leurs récits à travers ce prisme.

$\mathrm{Au}$ fond de la scène à Cour, cinq chaises permettent aux acteurs de s'asseoir et écouter lorsqu'ils ne jouent pas, et deviennent aussi des éléments de décors pour certaines scènes, comme la scène du divan, critique drôle et acérée de la prise en charge psychologique des New-Yorkais :

\section{Le divan}

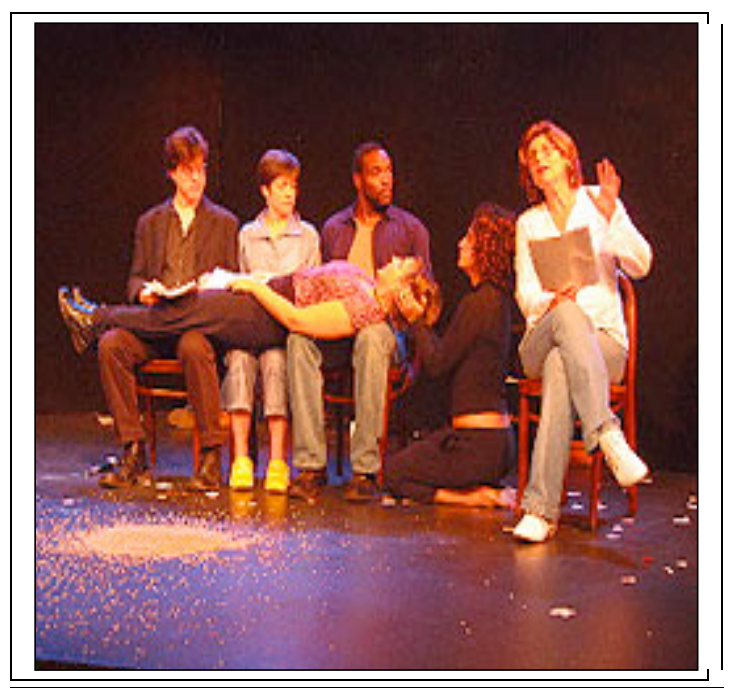

Trois acteurs s'assoient sur des chaises et deviennent le divan sur lequel la patiente s'allonge. Un autre soutient la tête de la patiente.

LA THÉRAPEUTE. Laissez-moi deviner? Vous avez une histoire du 11 septembre dont vous voulez me parler.

LA PATIENTE. Je crois que ça m'aidera à explorer mes sentiments, si je vous raconte mon histoire...

LA THÉRAPEUTE. Comme c'est original! Donc vous voulez explorer vos sentiments sur le 11 septembre.

\footnotetext{
${ }^{17}$ Samuel Beckett, « Texts for Nothing, » in The Complete Short Prose 1929-1989, New York, Grove Press, 1995,
} p.116. 
Divan Un. Refoule-les.

DIVAN DEUX. Renie-les.

DIVAN TROIS. Mets-les sur Internet.

[...]

LA THÉRAPEUTE. Etiez-vous dans les tours?

LA PATIENTE. Non.

LA THÉRAPEUTE. Avez-vous perdu quelqu'un?

LA PATIENTE. Non.

LA THÉRAPEUTE. Etiez-vous au moins en-dessous de Canal Street quand ça s'est produit?

LA PATIENTE. Non.

LA THÉRAPEUTE. Qu'est-ce que je peux vous dire?

LA PATIENTE. Mon histoire n'est pas pertinente... Je n'arrive pas à respirer. [...]

LA THÉRAPEUTE. Ok, il est clair que vous souffrez du syndrome de stress post-traumatique.

Je vous conseille de prendre ça pour dormir, ça pour vous réveiller, et ça pour travailler. Si

vous voulez bien signer ces papiers, vous deviendrez immédiatement une authentique

statistique du 11 septembre. Félicitations. ${ }^{18}$

\section{Retrouver le témoin par le chœur}

Tout au long de la session du thérapeute, les acteurs tenant lieu de canapé commentent l'échange et l'action est encadrée, voire parasitée, par des commentaires extérieurs. Ceux qui ne jouent pas réagissent, ils sont présents et à l'écoute, membres d'une communauté nécessaire à la parole. L'organisation de l'espace porte les traces de la choralité des tragédies grecques : sans être véritablement un espace de chœur séparé du reste de la scène, les cinq chaises permettent aux acteurs qui ne jouent pas de regarder les scènes tout en restant à vue. À aucun moment un acteur ne sort ; ils rentrent tous ensemble et restent jusqu'à la fin. Pour Jack Saul, " la communauté est un soutien et une protection lors de l'évocation de l'expérience émotionnelle du trauma ${ }^{19}$.» Tous les acteurs sont membres du chœur, il n'y a pas de protagoniste sinon celui qui se démarque pour parler à un moment donné puis revient et laisse la parole à d'autres. Les acteurs n'interviennent pas en tant qu'ensemble, ils ont chacun une voix individuelle, mais par leur positionnement dans l'espace, autour de celui qui parle, autour de la parole, ils forment un chœur disparate qui soutient ou remet en question la parole proférée sur scène. Jack Saul remarque que

le théâtre élargit le sens que les gens ont de la signification d'un évènement. Parmi ceux qui ont vu la pièce, beaucoup ont affirmé que c'était la première fois qu'ils avaient été capables de toucher du doigt certaines des émotions qu'ils avaient ressenties en conséquence du 11 septembre sans se sentir submergés ou re-traumatisés ${ }^{20}$.

La choralité est importante pour plusieurs raisons. D'abord parce qu'elle apporte une diversité et des perspectives à l'évènement, par exemple par le personnage de Sandro, et aussi parce qu'elle permet le surgissement du rituel, et notamment le rituel de deuil qui est celui qui a manqué le plus aux survivants du 11 septembre. Au cours de la pièce, l'évocation la plus forte est celle des corps. Il y a un réel besoin de parler des corps morts, ceux que l'on n'a jamais retrouvés, ceux que l'on a retrouvés sans pouvoir les identifier, etc. Samuel parle du corps de son petit-fils, Jérémy (il est aussi important de nommer), dont on a retrouvé seulement un bout. Cela fut suffisant pour organiser des funérailles. La révérende parle d'un corps retrouvé en décembre avec un portefeuille et une photo du visage du mort : «Normalement, ils n'ont plus de visages; ils ne sont pas reconnaissables ${ }^{21}$. » La confrontation avec un visage lui a fait une forte impression car elle rend impossible l'oubli de l'humanité des corps que les travailleurs dégagent. Elle précise, à l'intention du public et des personnages sur scène, que tout ce qui ressemblait de près ou de loin à des restes humains a été

\footnotetext{
${ }^{18}$ Downtown Community Resource Center Theater Group, op. cit. Extrait.

19 Ariane Zaytzeff, Interview avec Jack Saul, New York, 18/02/2008.

${ }^{20}$ Jack Saul, « Trauma and Performance : Constructing Meaning after Tragedy, Theater of Witness in Lower Manhattan Post 9/11 ». Présenté lors du Trauma and Research Net, Hamburg Institute for Social Research, St. Mortiz, 2006. p.41.

${ }^{21}$ Downtown Community Resource Center Theater Group, op. cit. Extrait.
} 
béni, comme pour les rassurer sur l'existence d'un rituel, d'une prise en charge des morts, d'une reconnaissance.

La pièce a été jouée trois fois en août 2003, puis de nouveau trois fois en septembre 2003, avec quelques changements inspirés des discussions avec le public. Celles-ci ont été enregistrées et préservées. Voici un exemple de questions/réponses :

\begin{abstract}
SPECTATEUR 1. Pourquoi faites-vous ce genre de théâtre ? Est-ce qu'il s'agissait de théâtre ou de thérapie?

ACTEUR. Pourquoi faire ce genre de théâtre ? L'idée originelle était de permettre une sorte de guérison ou réparation, non pas thérapeutique mais un processus public de réparation. Le temps que nous obtenions des subventions, beaucoup de temps avait passé. Les questions qui ressortaient quand nous avons commencé à travailler sur la pièce concernaient la signification de l'évènement et la façon dont il était utilisé pour plusieurs buts. Ce n'était plus centré sur ce que les gens ont vécu et la façon dont cela a affecté leurs vies.

SPECTATEUR 2. Ça m'a semblé être un bout de journal intime dans une histoire très longue. La pièce est l'expression des histoires de ces gens, et finalement cela deviendra les histoires d'autres personnes.

SPeCtATEUR 3. Pour moi, je n'ai jamais entendu ce genre d'histoires, ni dans les médias ni dans aucune autre forme d'expression publique ${ }^{22}$
\end{abstract}

L'équilibre entre théâtre et thérapie est à noter, et il est certain qu'un des buts de la pièce était d'offrir un forum public d'écoute et de partage à ceux qui avaient vécu le 11 septembre de très près et étaient toujours très affectés par ses conséquences. Mais il ne s'agissait pas de thérapie à proprement parler, et l'inspiration principale de la démarche théâtrale vient plutôt de l'histoire orale. En effet, pour Jack Saul, le silence qui est souvent imposé aux témoins après une catastrophe est un « traumatisme après le traumatisme ${ }^{23}$ », et c'est à cela en particulier qu'il voulait répondre. Le but de l'histoire orale est d'aller chercher les récits des groupes qui ont été marginalisés et dont les expériences ont été disqualifiées. Créer une pièce de théâtre signifiait offrir un espace public où ces histoires pourraient être entendues différemment. Abigail Gampel reconnaît que la pièce a eu du mal à toucher le public ciblé, c'est-à-dire les communautés du sud de Manhattan. Les membres de ces communautés sont venus en moins grand nombre que ce que le groupe espérait, et cela est peutêtre dû au fait qu'au moment des représentations, le DCRC avait été fermé si bien que la pièce s'est jouée à Here, un théâtre sur Dominick Street et la sixième avenue, à la limite nord de ce que l'on considère le sud de Manhattan. Cela est peut-être aussi le signe que la pièce a réussi à ouvrir l'expérience du 11 septembre à une communauté plus grande que celle du seul sud de Manhattan. En incluant les récits d'habitants de Chinatown et de Harlem, le groupe a étendu l'impact des attentats à tous les New-Yorkais, et avec ceux du Chili et de l'Italie, il a montré l'intégration de l'évènement dans une histoire qui le dépasse. Au fur et à mesure des interviews et des recherches, les acteurs ont découvert que les frontières du quartier sud de Manhattan devenaient floues, se mêlant à la ville dans son entier, au pays, au monde. En donnant forme à la pièce, ils voulu permettre à l'action du témoin de révéler et démêler les connexions existantes entre chaque situation.

Le dixième anniversaire du 11 septembre est l'occasion d'un foisonnement de travaux théâtraux, dont la 9/11 Play Series au Gerald W. Lynch Theater à Manhattan du 7 au 11 septembre, qui présentera cinq pièces sur le thème de la terreur, et la reprise de Return to the Upright Position à la Michigan State University. Il est question de reprendre Everything is Back to Normal dans les mois qui suivent. Abigail Gampel considère que le travail n'est pas fini et ne le sera probablement jamais : il y a plus de personnes à interviewer, plus de réactions à intégrer, et surtout cela serait l'occasion de retravailler la pièce avec des acteurs de la première heure et des nouveaux venus, la probabilité de réunir toute l'équipe d'origine étant faible. Le laps de temps écoulé amènerait aussi de nouvelles questions et de nouveaux angles d'approche à explorer. Contrairement au projet d'archive,

\footnotetext{
22 Jack Saul, « Trauma and Performance » art. cit. pp. 22-23.

${ }^{23}$ Id. p. 13.
} 
le travail théâtral n'est pas un travail de conservation mais d'actualisation, et comme le précise un des acteurs dans le bord de scène cité ci-dessus, le processus créatif s'adapte aux questions du moment. Témoigner du 11 septembre c'est témoigner de l'évènement dans le passé ainsi que dans ses métamorphoses et son extension dans le présent, dans les vies quotidiennes. Reprendre la pièce signifierait donc retourner auprès des diverses communautés touchées par les attentats pour constater leurs situations, préoccupations et attentes, et serait l'occasion d'identifier la portée du 11 septembre aujourd'hui et les questions que l'évènement et ses conséquences soulèvent à présent.

Samuel Beckett, « Texts for Nothing, » in The Complete Short Prose 1929-1989, New York, Grove Press, 1995.

Augusto Boal, Theatre of the Oppressed, Londres, Pluto Press, 1979.

George W. Bush, « National Day of Prayer and Remembrance for the Victims of the Terrorist Attacks on September 11, 2001, 》 url=http://www.whitehouse.gov/news/releases/2001/09/20010913-7.html, consulté le 25/05/2008.

-, «Remarks Upon Arrival, » url=http://www.whitehouse.gov/news/releases/2001/09/200109162.html, consulté le 25/05/2008.

Downtown Community Resource Center Theater Group, Everything's Back to Normal in New York City: Below Canal, A Work in Progress. Texte des représentations des 28, 29 et 30 septembre 2003.

S. Felman. et D. Laub, Testimony : Crises of Witnessing in Literature, Psychoanalysis and History, New York, Routledge, 1992.

Marc Nichanian, "Catastrophic Mourning » in Loss: The Politics of Mourning, Eng, D.L. and Kazanjian, D. (ed.), Berkeley, University of California Press, 2003, pp.125-147.

Steven Reisner, «Private Trauma/Public Drama : Theater as a Response to International Political Trauma » in Public Sentiments, Ann Cvetkovich et Ann Pellegrini (ed.), Special Issue of S\&F Online 2.1 (2003), url=http://www.barnard.edu/sfonline/ps/reisner.htm, consulté le 06/05/2010.

Jack Saul, « Trauma and Performance: Constructing Meaning after Tragedy, Theater of Witness in Lower Manhattan Post 9/11. » Présenté au Trauma and Research Net, Hamburg Institute for Social Research, St. Mortiz, 2006.

-, " Promoting Community Resilience in Lower Manhattan After September 11, 2001 », in AFTA Monograph Series, Winter 2007.

-, "International Trauma Studies Program », in The New Humanitarians : Inspiration, Innovation and Blueprints for Visionaries, Chris E. Stout (ed.), Londres, Praeger, 2008, pp. 209-230.

Diana Taylor, "Lost in the field of vision : Witnessing September 11 » in The Archive and the Repertoire : Performing Cultural Memory in the Americas, Durham, Duke University Press, 2003. Ariane Zaytzeff, Interview avec Jack Saul, New York, 18/02/2008.

-, Interview avec Abigail Gampel, New York, 23/05/2011.

\section{Pour citer ce document}

Ariane Zaytzeff, «Une expérience de théâtre témoin», Agôn [En ligne], Dossiers, HS n $1:$ Mettre en scène l'événement, Mémoire de l'événement : le cas du 11 septembre au théâtre, mis à jour le : 26/08/2011, URL : http://agon.ens-lyon.fr/index.php?id=1772. 